\title{
Deficient Herpes Simplex Virus-Induced Interferon- $\alpha$ Production by Blood Leukocytes of Preterm and Term Newborn Infants
}

\author{
BRITTA CEDERBLAD, TOMAS RIESENFELD, AND GUNNAR V. ALM \\ Interferon Laboratory [B.C., G.V.A.] and Department of Pediatrics [T.R.], Uppsala University and Division of \\ Immunology [G.V.A.], Department of Veterinary Microbiology, Swedish University of Agricultural Sciences, \\ Uppsala, Sweden
}

\begin{abstract}
The ability of peripheral blood mononuclear cells (PBMC) from newborn infants, gestational age 24 $42 \mathrm{wk}$, to produce interferon- $\alpha($ IFN- $\alpha)$ on the first day after birth was studied in vitro. Human amnion cells (WISH) coated with herpes simplex virus type I and fixed by glutaraldehyde were used as IFN- $\alpha$ inducers. Individual IF $-\alpha$ producing cells (IPC) among PBMC were determined by an immunoplaque assay. The frequency of IPC was low in all premature $(\leq 36 \mathrm{wk})$ infants (median 0.3 IPC $/ 10^{4} \mathrm{PBMC}$, range $0.0-2.6$ ), and significantly higher (median 2.0 IPC/104 PBMC, range 0.0-16.4) in term infants $(>37 \mathrm{wk})$. The frequencies were lower in both groups of infants than in adults (7.3 IPC/10 $10^{4}$ PBMC, range 2.023.7). When a conditioned medium from cultures of herpes simplex virus type I-stimulated PBMC from adults was added to the IFN induction cultures, the frequencies of IPC increased in PBMC from both preterm and term infants, and in the latter group did not differ significantly from adult levels. The median production of IFN- $\alpha$ per IPC was $1.1 \mathrm{U}$ (range 0.0-2.8) in premature infants, $1.0 \mathrm{U}$ (range 0.0-8.8) in term infants and 3.2 $U$ (range 1.5-8.0) were decreased, a decline of IPC frequencies occurred. This decline was more marked and started at higher PBMC concentrations in infants than in adults, and was prevented by addition of conditioned medium from herpes simplex virus type I-stimulated cultures of PBMC from adults. The results suggest that $\mathrm{PBMC}$ of preterm infants on the first day after birth are deficient both with respect to the proportion of actual IPC and to accessory mechanisms necessary for a normal IFN- $\alpha$ response. In contrast, IPC frequencies in term infants approach levels of adults, but accessory functions may still be deficient. (Pediatr Res 27:7-10, 1990)
\end{abstract}

\section{Abbreviations}

FBS, fetal bovine serum

HSV, Herpes simplex virus type 1

IFN, interferon

IPC, IFN- $\alpha$-producing cell

PBMC, peripheral blood mononuclear cells

Viral infections are relatively frequent and often more severe in newborn, especially preterm, infants than in adults. This

Received June 8, 1989: accepted August 31, 1989

Correspondence and reprint requests Britta Cederblad, Interferon Laboratory, Biomedical Center, Box 582, 75123 Uppsala, Sweden.

Supported by grants from the Swedish Medical Research Council, the Samaritan Foundation. Stockholm, and the Gillberg Foundation. Uppsala. decreased resistance to viruses, such as the clinically important herpes viruses (1), may be explained by an immaturity of the immune system (2-4). Also deficiencies in the IFN system, which contributes to the natural immunity to viruses $(5,6)$, may be of importance. However, the results of several previous studies of the IFN system indicate that at least the ability of PBMC from term newborn infants to produce IFN- $\alpha$ is similar to that of adults (7-11). Systematic studies of PBMC of preterm newborn infants $(\leq 36 \mathrm{wk}$ ) however, have not been reported.

In vitro, PBMC from adults can be induced to produce IFN- $\alpha$ by stimulation with HSV-infected glutaraldehyde-fixed cells (12, 13). The IPC among PBMC can be enumerated by an immunoplaque technique, for example (14). The IPC constitute only a small but highly efficient subpopulation among PBMC. The IPC lack both NK cell activity and markers of typical T or B lymphocytes and monocytes/macrophages (15-17). The in vitro IFN- $\alpha$ production by PBMC from adults may require as yet unidentified soluble accessory factors released by PBMC (18). This is suggested by the exceptionally low IFN- $\alpha$ responses at low PBMC concentrations. The lowered response can be restored by addition of conditioned medium from HSV-stimulated PBMC from adults.

In our study we examined the HSV-induced IFN- $\alpha$ production by PBMC, obtained directly after birth from children born at 24-42 wk of gestation. The frequencies of IPC were determined by the immunoplaque technique, and the mean amounts of IFN$\alpha$ produced per IPC were calculated. Also the influence of various concentrations of PBMC on the IPC response and its degree of enhancement by soluble factors released by HSV-activated PBMC from adults was studied.

\section{MATERIALS AND METHODS}

Blood samples. Samples were drawn from 14 consecutive preterm infants, gestational age 24-36 wk, birth wt 520-2360 g, and 17 term infants, gestational age 37-42 wk, birth wt 2730$4470 \mathrm{~g}$. All birth wt were appropriate for gestational age for Swedish infants. Of the preterm infants, 12 were treated with continuous positive airway pressure or ventilator because of respiratory disturbances. One of the preterm infants later died in a cerebral hemorrhage, the other infants survived. In all infants born before 32 wk of gestation, an umbilical artery was catheterized directly after birth as a routine clinical procedure. In infants born after $32 \mathrm{wk}$ of gestation, the clinical condition (respiratory disturbances) required umbilical arterial or, in two patients (rhesus immunization), umbilical venous catheterization. In 11 of the term infants umbilical cord blood was taken from the placental end of the cut cord. In these cases the infants were all healthy. Adult venous blood was obtained from 11 donors. The study was approved by the Ethical Committee of the Medical Faculty, Uppsala University. 
The blood samples were always drawn directly after umbilical catheterization into heparinized tubes (Vacutainer, Becton Dickinson, Rutherford, $\mathrm{NJ}$ ) and usually processed within $1 \mathrm{~h}$ after collection. Occasional samples were stored for at most $16 \mathrm{~h}$ at $+4^{\circ} \mathrm{C}$. No difference was observed in the IFN- $\alpha$ producing capacity of PBMC from adults prepared from fresh blood or from blood stored for $24 \mathrm{~h}$ at $+4^{\circ} \mathrm{C}$ (data not shown). The PBMC were separated on Ficoll-Hypaque gradients (Pharmacia, Uppsala, Sweden) and washed twice in PBS. The PBMC were resuspended in ice-cold FBS (Cytosystems, Castle Hill, N.S.W., Australia) containing $10 \%$ dimethyl sulfoxide, frozen at $-80^{\circ} \mathrm{C}$ for $4-24 \mathrm{~h}$, and then stored in liquid nitrogen until tested for IFN- $\alpha$ production.

Induction of IFN. The frozen PBMC were rapidly thawed, washed once and resuspended in RPMI 1640 medium, supplemented with L-glutamine ( $2 \mathrm{mM})$, penicillin $(60 \mu \mathrm{g} / \mathrm{mL})$, streptomycin $(100 \mu \mathrm{g} / \mathrm{mL})$, HEPES $(20 \mathrm{mM})$, and $10 \% \mathrm{FBS}$. The cells from all samples were diluted to a concentration of $2 \times 10^{6} /$ $\mathrm{mL}$, if not otherwise indicated.

The IFN-induction was carried out as previously described $(14,18)$. In short, human WISH cells were seeded in 96-well microtiter plates and fixed with glutaraldehyde. The plates were then incubated with $\mathrm{HSV}$ for $6 \mathrm{~h}$ at $37^{\circ} \mathrm{C}$, then again fixed, blocked with glycine, and finally rinsed with PBS. The PBMC were added to the induction wells in triplicates $(0.1 \mathrm{~mL} /$ well $)$, and incubated for $8 \mathrm{~h}$ at $37^{\circ} \mathrm{C}$ and $7 \% \mathrm{CO}_{2}$ in air.

The induction of IFN was carried out also in the presence of $25 \%$ of a conditioned medium. The conditioned medium was collected from cultures of $\mathrm{T}$ cell-depleted nonadherent PBMC from adults, stimulated for $6 \mathrm{~h}$ by HSV-infected WISH cells in 24-well plates. Such conditioned medium has an enhancing effect on the IFN- $\alpha$ response of PBMC from adults, especially at suboptimal culture conditions, without itself inducing IFN- $\alpha$ (18).

Immunoplaque assay. The detection of individual IPC was performed as previously described $(14,18)$. In brief, nitrocellulose bottomed 96-well Millititer-HA plates (Millipore Co., Bedford, MA) were coated with bovine anti-IFN- $\alpha$ antibodies (AS 94, Wellcome Research Foundation, Beckenham, UK), fixed with glutaraldehyde, and washed with PBS. The induced PBMC were suspended after $8 \mathrm{~h}$ in the induction cultures and transferred in a one-to-one well fashion to the millititer plates. After overnight incubation at $37^{\circ} \mathrm{C}$, the plates were incubated with the murine monoclonal anti-IFN- $\alpha$ antibody LO 22 (provided by Dr. K. Berg, Copenhagen, Denmark) $(19,20)$, peroxidase conjugated goat-antimouse IgG antibodies (Jackson ImmunoResearch, Avondale, PA), and the substrate diaminobenzidine, in sequence with intervening washes. The stained plaques in each well were counted under a macroscope.

Control experiments showed that the IPC frequencies were the same in previously frozen PBMC as in fresh PBMC from both infants and adults.

IFN assay. The antiviral activity was measured as inhibition of the cytopathic effect of vesicular stomatitis virus in WISH cell cultures (21). The National Institute of Health reference leukocyte IFN- $\alpha$ GA-23-902-530 was used to calibrate our own internal laboratory standard. The quantity of IFN produced per IPC was then calculated by dividing the amount of IFN per culture with the number of IPC detected in parallel induction cultures.

Statistical analysis. Data are expressed as medians and range for each group. Differences between groups were analyzed using the Mann-Whitney U test or, for paired observations, the Wilcoxon signed rank test. Groups were considered to differ significantly when $p$ was less than 0.05 .

\section{RESULTS}

IF $N$ - $\alpha$ response in newborn infants of different gestational age. Figure 1 shows that the frequencies of IPC were low in PBMC

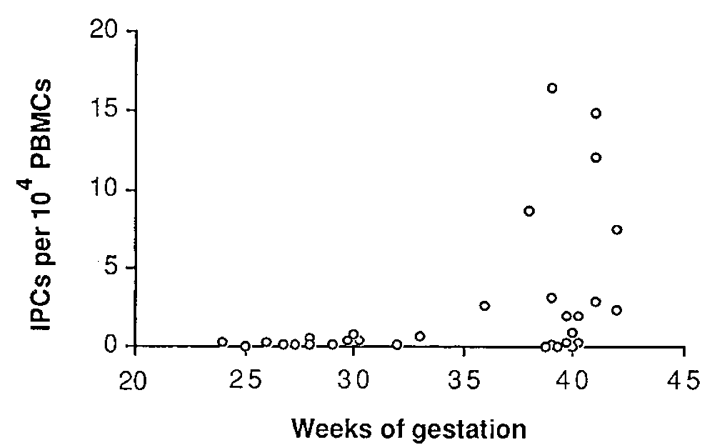

Fig. 1. Frequencies of IPC in cultures of PBMC obtained from 31 infants born at various weeks of gestation. PBMC $\left(2 \times 10^{6} / \mathrm{mL}\right)$ were induced to produce IFN- $\alpha$ by glutaraldehyde-fixed HSV-infected WISH cells for $8 \mathrm{~h}$. The IPC-frequencies were determined by the immunoplaque assay.

Table 1. Frequencies of IPC in PBMC from preterm and term infants compared to those in $P B M C$ from adults*

\begin{tabular}{ccc}
\hline & \multicolumn{2}{c}{ IPC/104 PBMC } \\
\cline { 2 - 3 } PBMC source & Without CM & With CM \\
\hline Preterm infants $(\mathrm{P}) \dagger \dagger$ & 0.3 & 1.0 \\
& $(0.0-2.6, n=14)$ & $(0.1-3.5, n=12)$ \\
Term infants (T) & 2.0 & 4.2 \\
& $(0.0-16.4, n=17)$ & $(0.0-35.0, n=16)$ \\
Adults (A) & 7.3 & 8.5 \\
& $(2.0-23.7, n=11)$ & $(2.3-23.2, n=11)$ \\
\hline
\end{tabular}

* PBMC $\left(2 \times 10^{6} / \mathrm{mL}\right)$ were induced with or without $25 \%$ of conditioned medium (CM) (see "Materials and Methods") on glutaraldehydefixed HSV-infected WISH cells for $8 \mathrm{~h}$. The IPC-frequencies were subsequently determined by the immunoplaque assay. Figures are medians. Range and number of individuals tested are given in parentheses.

$\dagger$ Significance levels as determined by the Wilcoxon signed rank test: Preterm infants without $C M$ versus preterm with $C M$ (P versus $P+C M)$ : $p<0.005$; $\mathrm{T}$ versus $\mathrm{T}+\mathrm{CM}: p<0.005$.

$\ddagger$ Significance levels as determined by the Mann-Whitney $\mathrm{U}$ test: $\mathrm{P}$ versus T: $p<0.05$; $\mathrm{P}$ versus $\mathrm{A}: p<0.001$; T versus $\mathrm{A}: p<0.05$; all infants versus $\mathrm{A}: p<0.001 ; \mathrm{P}+\mathrm{CM}$ versus $\mathrm{T}+\mathrm{CM}: p<0.02 ; \mathrm{P}+\mathrm{CM}$ versus $\mathrm{A}+\mathrm{CM}: p<0.001$.

collected from all the infants born before $36 \mathrm{wk}$ of gestation. In most $(11 / 18)$ infants born at 36 wk of gestation or later, higher IPC frequencies were seen. As summarized in Table 1, the IPC frequency in the group of preterm infants was significantly lower than in term infants $(p<0.05)$. The IPC frequencies in both groups, as well as in the combined group of infants, were significantly lower than in adults $(p<0.001, p<0.05$, and $p<0.001$, respectively).

No difference was observed between the IPC frequencies of PBMC from cord blood or from blood collected by cannulation of umbilical veins or arteries of term infants (results not shown).

The quantity of IFN produced per IPC was calculated from the concentration of IFN in the culture supernatants after $24 \mathrm{~h}$ of induction, divided by the number of IPC per culture. Each detected IPC corresponds in preterm infants to $1.1 \mathrm{U}$ IFN (range $0.0-2.8, n=7)$, in term infants to $1.0 \mathrm{U}$ (range $0.0-8.8, n=8$ ), and in adults to $3.2 \mathrm{U}$ (range $1.5-8.0, n=10$ ). The IFNproduction per IPC of both preterm infants and the combined group of infants, but not term infants solely, were significantly lower than that of adults $(p<0.01)$.

Effect of conditioned medium on induction of IPC. The induction cultures were supplemented with conditioned medium from cultures of HSV-stimulated PBMC from adults. Such condi- 
tioned medium at a concentration of $25 \%$ increased the IPC frequencies significantly in cultures of PBMC derived from both preterm and term infants $(p<0.005)$, but not from adults (Table 1). However, the net increase in IPC frequencies in the preterm infants was small $\left(0.7 \mathrm{IPC} / 10^{4} \mathrm{PBMC}\right)$. Despite addition of conditioned medium, significant differences still remained between frequencies of IPC of preterm and term infants $(p<0.02)$, between preterm infants and adults $(p<0.001)$, but not between IPC frequencies of term infants and adults.

The results described above were obtained at a concentration of $2 \times 10^{6} \mathrm{PBMC} / \mathrm{mL}$. When sufficient amounts of PBMC were available, the IFN- $\alpha$ response was evaluated at different concentrations of PBMC in the induction cultures, with or without addition of conditioned medium. The results from these dilution experiments are given in Figures $2 A$ and $B$, respectively. The gradual decrease in frequencies of IPC with decreasing concentrations of PBMC was more pronounced in the two groups of infants than in adults (Fig. $2 \mathrm{~A}$ ). Thus, the median frequencies of IPC at $0.5 \times 10^{6} \mathrm{PBMC} / \mathrm{mL}$ of preterm and term infants were only 25 and $12 \%$, respectively, of the peak IPC frequencies at 2 $\times 10^{6} \mathrm{PBMC} / \mathrm{mL}$, compared to $74 \%$ in adults. Consequently, the differences between IPC frequencies of term infants and adults are more prominent at $0.5 \times 10^{6}$ than at $2 \times 10^{6} \mathrm{PBMC} /$ $\mathrm{mL}(p<0.002$ and $<0.05$, respectively).

The conditioned medium largely abrogated the decrease in frequencies of IPC at lowered concentrations of PBMC (Fig. 2B). Consequently, the effect of conditioned medium, i.e. the increased frequencies of IPC, were most marked at the lowest concentrations of PBMC.
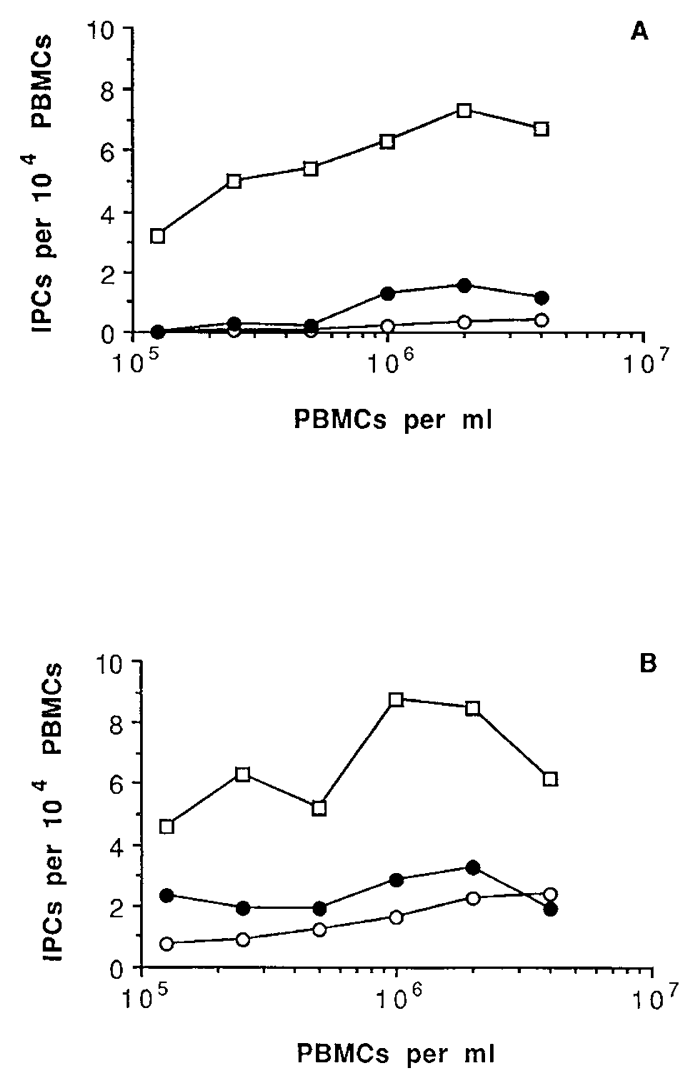

Fig. 2. Frequencies of IPC (medians) at different concentrations of PBMC in the induction cultures. Induction (see legend to Fig. 1) were performed in the absence $(A)$ or in the presence $(B)$ of $25 \%$ of conditioned medium from cultures of PBMC from adults stimulated for $6 \mathrm{~h}$ by glutaraldehyde-fixed HSV-infected WISH cells. PBMC from preterm $(\mathrm{O}$, $n=5$ in $A, n=4$ in $B)$ and term $(\bullet, n=10$ in $A, n=8$ in $B)$ infants, and from adults $(\square, n=11$ in $A$ and $B)$.

\section{DISCUSSION}

We could demonstrate that the IFN- $\alpha$ response of PBMC from newborn infants, especially preterm ones, was deficient compared to that of PBMC from adults. This was evident as lower frequencies of IPC and as lower quantities of IFN- $\alpha$ produced per cell after in vitro stimulation by glutaraldehyde-fixed HSVinfected WISH cells.

At high $\left(2 \times 10^{6}\right.$ cells $\left./ \mathrm{mL}\right)$ concentrations of PBMC obtained from term newborn infants, the HSV-induced IFN- $\alpha$ response was decreased but could be restored to levels not significantly different from adults by addition of conditioned medium from cultures of adult PBMC stimulated by HSV. Such conditioned medium contains soluble factors, possibly cytokines, which appear to be important cofactors in the normal HSV-induced IFN$\alpha$ response (18). Thus, given the appropriate culture conditions, including concentrations of PBMC high enough to yield sufficient amounts of cofactor(s), the IFN- $\alpha$ responses of PBMC from term infants might appear relatively normal. This is in line with results of others that indicate normal levels of IFN- $\alpha$ in culture medium of HSV-stimulated $(10,11)$ or Sendai virus stimulated $(7,22)$ PBMC from term infants.

At low concentrations of PBMC our results clearly demonstrate lower IFN- $\alpha$ responses in term infants than in adults. The gradual decrease in frequencies of IPC at decreasing concentrations of PBMC was more pronounced in PBMC from term infants than from adults. This phenomenon could largely be prevented by addition of the conditioned medium. Our results are therefore compatible with the hypothesis that the frequency of potential IPC in term infants may not differ significantly from that of adults, but their IFN- $\alpha$ responses are, at least in vitro, limited by a deficiency in the production of accessory cofactor(s).

For preterm infants, the frequencies of IPC among the PBMC were about $5 \%$, and the quantity of IFN produced per IPC about $30 \%$, of adult levels when induced at $2 \times 10^{6} \mathrm{PBMC} / \mathrm{mL}$. The $\mathrm{HSV}$-induced IFN- $\alpha$ response in preterm infants was not only lower than in the adults but also significantly lower than in term infants, indicating a more profound defect in the IFN- $\alpha$ response in preterm infants. As the increase in frequencies of IPC caused by addition of conditioned medium was small in preterm infants, we suggest that their deficiency resides both in potential IPC and in the accessory mechanism(s).

It is interesting to note that a marked improvement in the IFN- $\alpha$ producing ability occurred in the infants born after gestational wk 36, marking the end of the preterm period. This change may therefore be part of a programed normal adaptation of the immune system to extrauterine life.

Our observations suggest that newborn infants, especially preterm ones, are deficient in their IFN- $\alpha$-producing capacity, at least when induced in vitro by HSV-infected cells. This defective IFN production may be one cause of the relatively high frequency, and the more severe course, of viral infections in newborn infants, particularly in preterm ones. It is therefore important to further explore the relevance of our findings to the in vivo situation.

Acknowledgments. The authors thank Anne Riesenfeld, AnnaLena Johansson, and Lisbeth Fuxler for excellent technical assistance.

\section{REFERENCES}

1. Whitley RJ, Nahmias AJ, Visintine AM, Fleming CL, Alford CA 1980 The natural history of Herpes simplex virus infection of mother and newborn. Pediatrics 66:489-494

2. Kohl S 1984 The immune response of the neonate to Herpes Simplex Virus Infection. In: Rouse BT, Lopez C (eds) Immunobiology of Herpes Simplex Virus Infection. CRC Press, Boca Raton, FL, pp 121-129

3. Wilson CB 1986 Immunological basis for increased susceptibility of the neonate to infection. J Pediatr $108: 1-12$ 
4. Sullender WM, Miller JL, Yasukawa LL, Bradley JS, Black SB, Yeager AS, Arvin AM 1987 Humoral and cell-mediated immunity in neonates with herpes simplex virus infection. J Infect Dis 155:28-37

5. Gresser I 1984 Role of interferon in resistance to viral infection in vivo. In: Vilcek J, De Mayer E (eds) Interferon 2: Interferon and the Immune System. Elsevier, Amsterdam, pp 221-247

6. Kirchner $H 1986$ The interferon system as an integral part of the defense system against infections. Antiviral Res 6:1-17

7. Ray CG 1970 The ontogeny of interferon production by human leukocytes. J Pediatr 76:94-98

8. Bryson YJ, Winter HS, Gard SE, Fisher TJ, Stiehm ER 1980 Deficiency of immune interferon production by leukocytes of normal newborns. Cell Immunol 55:191-200

9. Handzel ZT, Levin S, Dolphin Z, Schlesinger M, Hahn T, Altman Y, Schechter B, Shneyour A, Trainin N 1980 Immune competence of newborn lymphocytes. Pediatrics 65:491-496

10. Kohl S, Harmon MW 1983 Human neonatal leukocyte interferon production and natural killer cytotoxicity in response to herpes simplex virus. $J$ Interferon Res 3:461-463

11. Leibson PJ, Hunter-Laszlo M, Douvas GS, Hayward AR 1986 Impaired neonatal natural killer-cell activity to herpes simplex virus: decreased inhibition of viral replication and altered response to lymphokines. J Clin Immunol 6:216-224

12. Lebon P, Commoy-Chevalier MJ, Robert Galliot B, Chany C 1982 Different mechanisms for $\alpha$ and $\beta$ interferon induction. Virology 119:504-507

13. Capobianchi MR, Facchini J, Di Marco P, Antonelli G, Dianzani F 1985 Induction of alpha interferon by membrane interaction between viral surface and peripheral blood mononuclear cells. Proc Exp Biol Med 178:551-556
14. Rönnblom L, Cederblad B, Alm GV 1988 Determination of Herpes simplex virus induced alpha interferon-secreting human blood leukocytes by a filter immuno-plaque assay. Scand J Immunol 27:165-170

15. Lebon P, Bernard A, Boumsell L 1982 Human lymphocytes involved in $\alpha$ interferon production can be identified by monoclonal antibodies against cell surface antigens. C R Acad Sci Paris 295:79-82

16. Abb J, Abb H, Deinhardt F 1983 Phenotype of human $\alpha$-interferon producing leukocytes identified by monoclonal antibodies. Clin Exp Immunol 52:179184

17. Perussia B, Fanning V, Trinchieri G 1985 A leukocyte subset bearing HLA$\mathrm{DR}$ antigens is responsible for in vitro alpha interferon production in response to viruses. Nat Immun Cell Growth Regul 4:120-137

18. Cederblad B, Alm GV 1989 Infrequent but highly efficient interferon- $\alpha$ producing human mononuclear leukocytes induced by herpes simplex virus in vitro studied by immuno-plaque and limiting dilution assays. J Interferon Res (in press)

19. Berg K 1984 Identification, production and characterization of murine monoclonal antibody (LO 22) recognizing 12 native species of human alpha interferon. $J$ Interferon Res 4:481-489

20. Meager A, Berg K 1986 Epitope localization of a monoclonal antibody, LO 22 , with a broad specificity for interferon- $\alpha$ subtypes. J Interferon Res 6:729736

21. Ojo-Amaize EA, Salimonu LS, Williams AIO, Akinwolere OAO, Shabo R, Alm GV, Wigzell H 1981 Positive correlation between degree of parasitemia, interferon titres and natural killer cell activity in Plasmodium falciparum infected children. J Immunol 127:2296-2300

22. Cantell K, Strander H, Saxén L, Meyer B 1968 Interferon response of human leukocytes during intrauterine and postnatal life. J Immunol 100:1304-1309 